\title{
BMJ Open Assessing infant cognition in field settings using eye-tracking: a pilot cohort trial in Sierra Leone
}

\author{
Jukka M Leppänen, ${ }^{1}$ Julius Walker Butcher, ${ }^{2}$ Claire Godbout, ${ }^{3}$ \\ Kevin Stephenson (D) , ${ }^{4}$ D Taylor Hendrixson (D) , ${ }^{3}$ Stacy Griswold (D) ,5 \\ Beatrice Lorge Rogers, ${ }^{5}$ Patrick Webb (D) , ${ }^{5}$ Aminata S Koroma, ${ }^{6}$ Mark J Manary ${ }^{3}$
}

To cite: Leppänen JM, Butcher JW, Godbout C, et al. Assessing infant cognition in field settings using eyetracking: a pilot cohort trial in Sierra Leone. BMJ Open 2022;12:e049783. doi:10.1136/ bmjopen-2021-049783

- Prepublication history and additional supplemental material for this paper are available online. To view these files, please visit the journal online (http://dx.doi.org/10.1136/ bmjopen-2021-049783).

Received 02 February 2021 Accepted 14 January 2022

Check for updates

(C) Author(s) (or their employer(s)) 2022. Re-use permitted under CC BY-NC. No commercial re-use. See rights and permissions. Published by BMJ.

For numbered affiliations see end of article.

Correspondence to Dr Jukka M Leppänen; jukka.leppanen@utu.fi

\section{ABSTRACT}

Objectives To investigate the feasibility of eyetracking-based testing of the speed of visual orienting in malnourished young children at rural clinics in Sierra Leone.

Design Prospective dual cohort study nested in a clusterrandomised trial.

Setting 8 sites participating in a cluster-randomised trial of supplementary feeding for moderate acute malnutrition (MAM).

Participants For the MAM cohort, all infants aged 7-11 months at the eight sites were enrolled, 138 altogether. For controls, a convenience sample of all non-malnourished infants aged 7-11 months at the same sites were eligible, 60 altogether. A sample of 30 adults at the sites also underwent eye-tracking tests as a further control. Interventions Infants with MAM were provided with supplementary feeding.

Outcome measures The primary outcomes were feasibility and reliability of eye-tracking-based testing of saccadic reaction time (SRT). Feasibility was assessed by the percent of successful tests in the infants. Reliability was measured with intraclass correlation coefficients (ICCs). Secondary outcomes were mean SRT based on nutritional state as well as and changes in mean SRT after supplementary feeding of MAM children.

Results Infants exhibited consistent orienting to targets on a computer screen (>95\% of valid trials). Mean SRTs had moderate stability within visits (ICCs 0.60-0.69) and across the 4-week test-retest interval (0.53) in infants; the adult control group had greater SRT stability (within visit ICC=0.92). MAM infants had a trend toward higher adjusted SRT at baseline (difference=12.4 ms, 95\% Cl -2 to $26.9, p=0.09$ ) and improvement in SRT 4 weeks thereafter (difference $=-14 \mathrm{~ms}, 95 \% \mathrm{Cl}-26.2$ to -1.7 , $\mathrm{p}=0.025)$ compared with age-matched controls.

Conclusions The results demonstrate the feasibility of eye-tracking-based testing in a resource-poor field setting and suggest eye-tracking measures have utility in the detection of group level effects of supplementary feeding.

\section{INTRODUCTION}

Children who lack access to appropriate nutrition, care and environmental stimulation in early childhood are at heightened risk for compromised learning and cognition, as

\section{Strengths and limitations of this study}

- Extension of technologically advanced eye-tracking testing into underserved and severely resourcelimited settings.

- Robust testing of the reliability of the method employed.

- Repeated measurements collected for infants.

- Inclusion of two control groups, age-matched infants as well as adults, to investigate sources of variability in test results.

- Cohorts are small and included infants from a single district in rural Sierra Leone.

well as persistent cognitive, emotional and social problems in adulthood. ${ }^{1-7}$ Although anthropometry offers a crude assessment of development, it remains the dominant means of assessing undernutrition in its acute and chronic forms. Field-applicable tests of neurocognitive function could add a dimension to the assessment of childhood development and contribute to our understanding of the process and effects of acute and chronic malnutrition. ${ }^{8}$

As a first step toward investigating the acute neurocognitive effects of early childhood undernutrition, we examined the feasibility and measurement properties of visual orienting testing in infants in low-resource settings. The capacity to orient eyes to visual objects (eg, abrupt onset of a stimulus) and hold them on key sources of information (eg, faces) emerges rapidly during the first 6 months of life in infants, ${ }^{9-14}$ and provides a foundation for the child's ability to interact with the physical and social environment. ${ }^{15}$ No studies to date have tested whether measures of visual orienting are sensitive to short-term changes in neurocognitive status in individual children, but there is evidence of this utility in adults: the mean latency of visual orienting changes in response to mild 
traumatic brain injuries (concussion) and provides a reliable measure for monitoring neurocognitive recovery in individual persons over a few days' time. ${ }^{16}$ Studies of visual orienting in infants have shown positive associations between the speed of visual orienting at 4-7 months of age and performance IQ at 4 years of age,${ }^{17}$ as well as executive function at age $11 .{ }^{18}$ Reduced orienting to faces in infancy has been associated with a heightened risk for autism ${ }^{19}$ and propensity to social-behavioural problems at 4 years. ${ }^{20}$

Visual orienting testing in infants has benefited from the replacement of manual methods of eye movement coding with more automated methods of eye-tracking that use remote infrared light sources and cameras. ${ }^{21} 22$ The majority of studies examining infant visual orienting by automated eye-tracking come from high-resource laboratories in Western countries, but similar research has recently been expanded to assess visual orienting ${ }^{23-26}$ as well as other aspects of cognition in children in less studied populations in mid-income and low-income countries. ${ }^{27-29}$ These studies have shown that eyetracking can be performed in low-resource settings, typically at central healthcare facilities, ${ }^{26-28}$ although the use of a portable system at participants' homes in rural Vanuatu has also been reported. ${ }^{29}$ A study conducted in Malawi found no association between eye-tracking measures of visual orienting at 7-9 months and tests of cognitive and motor function at 18 months. ${ }^{26}$ However, positive correlations were found between eye-tracking measures of novelty preference (ie, preferential looking at a novel vs previously shown face) and conventional tests of cognitive function in 6-12 month-old Ugandan children. ${ }^{28}$

The psychometric properties of eye-tracking measures (ie, reliability) are critical to assess the utility of this method in monitoring short-term cognitive changes in children. To our knowledge, only one of the studies conducted in low-resource settings used repeated measurements to assess reliability, showing low correlation $(0.28)$ for the mean latency of visual orienting over a 2-month testretest interval. ${ }^{26}$ It is possible, however, that the reliability of the measurements was degraded by the low sampling rate of the eye tracker used $(60 \mathrm{~Hz})$ as well as the low number of valid test trials (3-8) per participant.

Direct comparisons of groups of malnourished and non-malnourished children on eye-tracking measures are lacking. Weight-for-age z-scores were not correlated with the latency of visual orienting in 9-month-old Malawian infants, but the association may have been affected by the low reliability of the eye-tracking measurements. A recent study conducted in rural Malawi showed no effects of a complementary feeding intervention (one egg per day) on the latency of visual orienting or novelty preference in 12-15 month-old Malawian children. ${ }^{30}$ However, a study in the USA reported faster mean saccadic reaction times (SRTs) at 4-13 months of age in infants whose mothers were supplemented with 930 versus $480 \mathrm{mg}$ of choline per day during the third trimester of pregnancy. ${ }^{31}$
We expanded previous eye-tracking studies in lowresource settings by examining whether mean SRTs provide a reliable measure of cognitive changes in acute malnutrition in infants living in villages in rural, SubSaharan Africa, where poor nutrition and growth failures are common. ${ }^{32}$ Mean SRT has been found to have moderate test-retest reliability in studies in high-resource settings $(r=0.65) .{ }^{33}$ Our aims were threefold. First, we examined the feasibility and measurement properties of SRT testing in infants in resource-poor settings by using a battery-powered eye tracker in a designated tent. Second, we tested the hypothesis that measures of visual orienting are associated with the nutritional status of the child, as demonstrated by slower SRT in malnourished children as compared with non-malnourished controls. Third, we examined whether visual orienting benefits from nutritional intervention.

\section{METHODS}

\section{Patient and public involvement}

Community leaders and local healthcare workers were engaged prior to the study to discuss the purpose of the study and nature of participation. Village leaders were also involved in site selection. Regular, open progress meetings with village leaders and local healthcare workers were held semiannually. A meeting for dissemination of results was held in August 2019 in Pujehun District.

\section{Participant recruitment and design}

Data for children were collected as a part of a larger intervention study comparing the cost-effectiveness of four supplementary foods in the treatment of moderate acute malnutrition (MAM) in Pujehun District, Sierra Leone, while data for adults were collected as part of a study assessing treatment approaches for MAM based on severity of wasting (figure 1) ${ }^{34}$ In the study setting, $29.9 \%$ of the population falls in the lowest quintile for income and $27.3 \%$ in the second lowest. The four foods intervention study was implemented in 29 Peripheral Health Units (PHUs), which were randomised to administer one of four supplementary foods to children 6-59 months of age diagnosed with MAM. Inclusion criteria were diagnosis of MAM, defined as mid-upper arm circumference (MUAC) $\geq 11.5 \mathrm{~cm}$ and $<12.5 \mathrm{~cm}$ without bipedal oedema, caregiver willingness to return to clinic every 2 weeks for assessment and ability of the child to consume the supplementary food as demonstrated at enrolment. Exclusion criteria included developmental disability, congenital malformation and current or recent (up to 1 month) enrolment in a separate supplementary feeding programme.

Children were evaluated by anthropometry every 2 weeks, and for a minimum of 2 weeks, until a programmatic outcome was reached. These included recovery, development of severe acute malnutrition, death, loss to follow-up or not recovered despite 12 weeks of supplemental feeding. They received one of the following foods: 


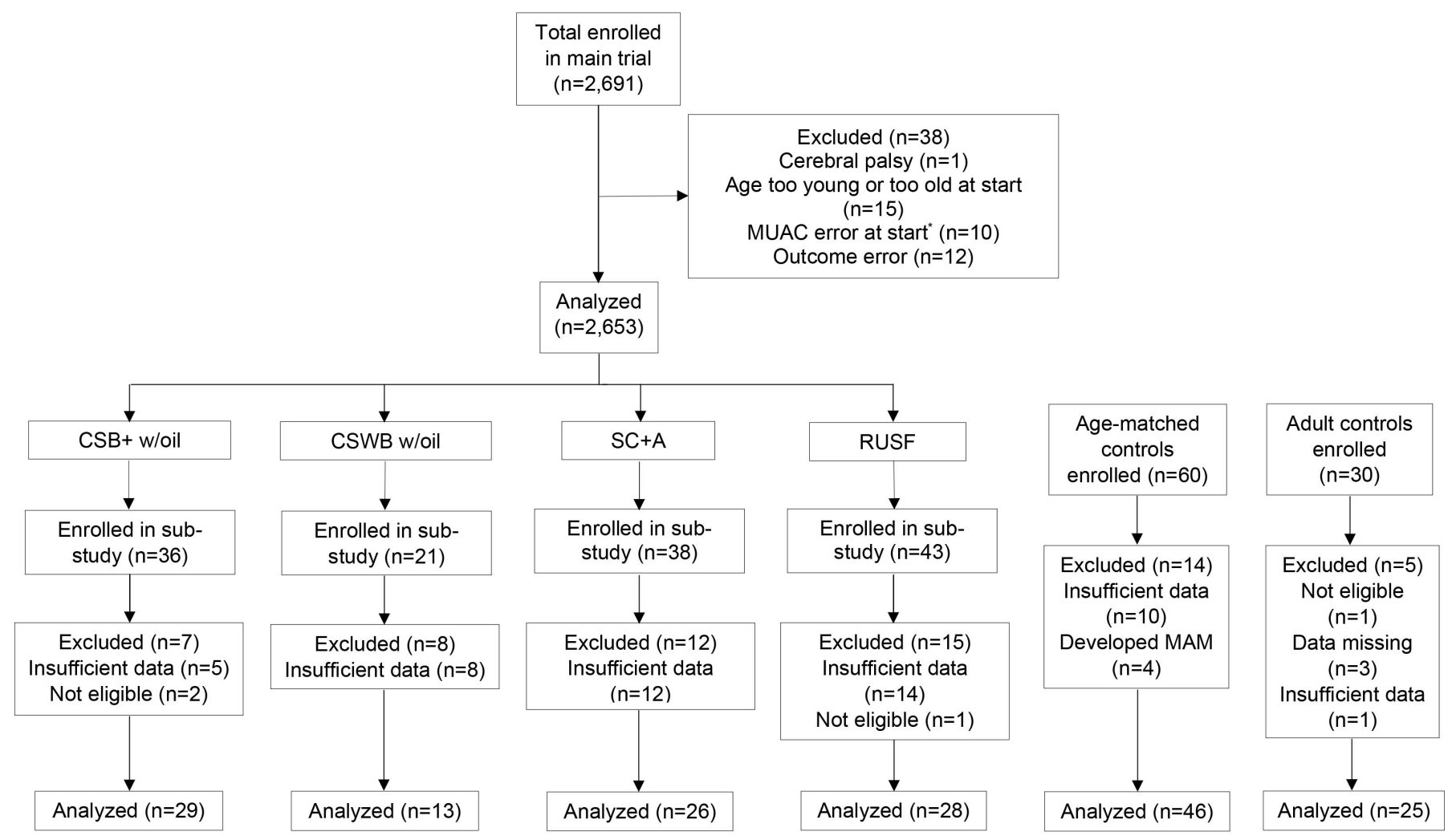

Figure 1 Trial flow diagram. CSB+, corn-soy blend plus; CSWB, corn-soy-whey blend; MAM, moderate acute malnutrition; MUAC, mid-upper arm circumference; RUSF, ready-to-use supplementary food; SC+A, super cereal plus with amylase. ${ }^{*}$ MUAC error at start was defined as mean of three MUAC measurements $\geq 12.5 \mathrm{~cm}$ or $<11.5 \mathrm{~cm}$.

(1) super cereal plus with amylase $(\mathrm{SC}+\mathrm{A})$; (2) corn-soy blend plus and fortified vegetable oil (CSB+w/oil); (3) corn-soy whey blend and fortified vegetable oil (CSWB w/oil) and (4) ready-to-use-supplementary food (RUSF) (online supplemental table 1 ). These foods were not formulated to improve cognition or visual function, but rather for nutritional recovery from MAM.

All infants 7-11 months of age with MAM who were enrolled into the parent study over a 10 -month period at the largest 8 of the 29 PHUs were also enrolled into this dual cohort substudy. The two largest PHUs for each study arm were chosen to participate in the substudy. Control children were recruited at these same 8 PHUs and over the same period of time. Controls were recruited sequentially and assessed via the same inclusion criteria previously listed, with the exception of having an MUAC $\geq 12.5$ $\mathrm{cm}$. Beyond the exclusion criteria of the parent study, three additional criteria were applied to both groups: (a) a known history of visual problems; (b) any neurological problem; (c) inadequate number of valid tests (ie, $<10$ valid SRTs/visit). In addition, controls were excluded if they developed malnutrition by the second clinic visit. A target sample size of 30 adults aged 18-40 from these same 8 PHUs were also recruited and enrolled sequentially as a convenience sample. Exclusion criteria were (a) a known history of visual problems; (b) any neurological problem; (c) inadequate number of valid tests (ie, $<10$ valid SRTs/visit).
All participants enrolled in the study were assessed by eye-tracking-based tests of visual orienting and by conventional observational tests of oculomotor function. Children were tested at enrolment and again 4 weeks later. The 4-week time period was chosen because all MAM children received 4 weeks of rations, even if they achieved recovery after 2 weeks.

The four foods MAM study received ethical approval from the Tufts University Health Sciences Institutional Review Board, the Washington University in St Louis School of Medicine Institutional Review Board and the Sierra Leone Ethics and Scientific Review Committee, while the MAM study in which adult controls were recruited received ethical approval from the Washington University and Sierra Leone review boards. The project was introduced to communities through an open meeting with the chiefs and village leaders. After all questions and comments were addressed, agreement was obtained for offering the study in their community. Following this, open community meetings were held detailing the study, usually attended by $25-50$ women, presumably mothers of the beneficiaries. Informed consent was obtained from all caregivers of participants with a private oral explanation of the procedure, risks and benefits. All beneficiary caregivers were illiterate, and signed or thumb-printed the consent form on behalf of their infants. The trial was registered at Clinicaltrials. gov (NCT03146897). 

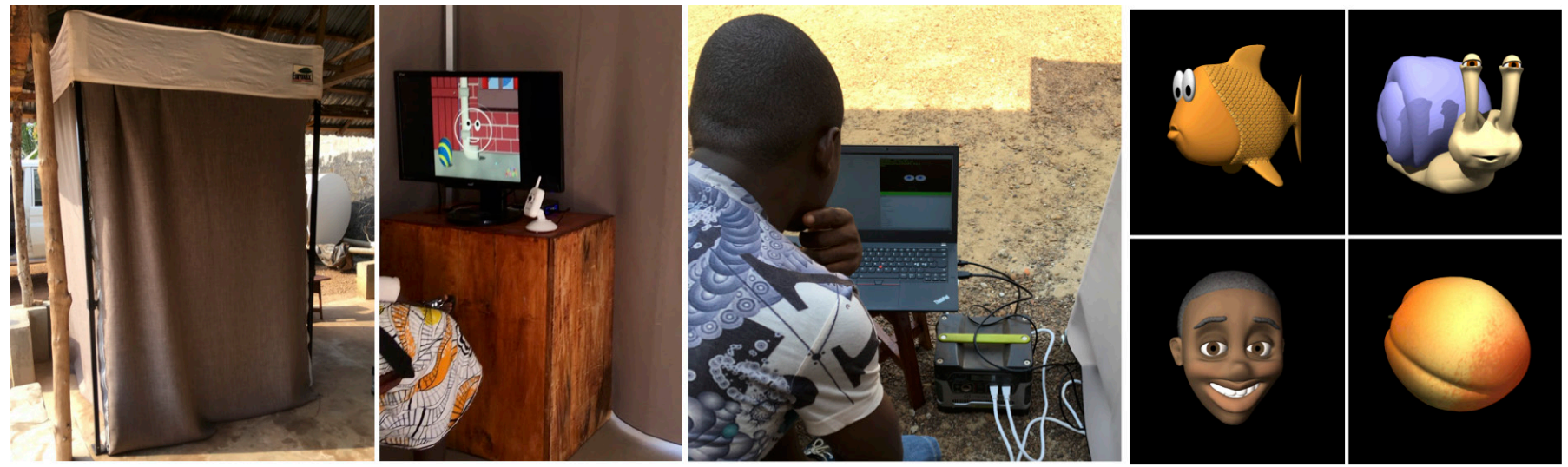

Figure 2 Left: a child positioned for eye-tracking assessment in a field laboratory tent, powered by a solar-chargeable battery. Middle: data collector monitored the assessment outside the laboratory via real-time visualisation of eye-tracking. Right: examples of stimuli used as targets in a test assessing saccadic reaction time.

\section{Eye-tracking testing}

\section{Setting and equipment}

Infants were assessed in a $1.5 \mathrm{~m}$ (width) $\times 1.5 \mathrm{~m}$ (length) $\times 2$ $\mathrm{m}$ (height) canopy tent with custom-made walls of monochromatic dimout fabric (figure 2). The tent was mounted in the vicinity of a PHU, typically on a covered outdoor porch area. The caregiver held the infant in a baby carrier so that the infant's eyes were facing forward and positioned at approximately $60 \mathrm{~cm}$ viewing distance from a 19-Inch Acer V196L LCD monitor (resolution: $1280 \times 1024$, refresh rate: $60 \mathrm{~Hz}$, response time: $5 \mathrm{~ms}$, manufacturer: Acer, San Jose, California, USA) and a screen-based Tobii X3-120 eye tracker (Tobii Technology, Stockholm, Sweden). The monitor and the eye tracker were placed on a wooden desk that was custom-made to be at suitable height for the participants in the sample age range. The infants were monitored during testing via a video recorder and a baby monitor. The video recorder (Sony HDR-CX240E, Sony Electronics, San Diego, California, USA) was mounted on top of the monitor. The baby monitor (Summer Infant, Woonsocket, Rhode Island, USA) was placed next to the monitor on the desk.

During the assessment, infants were presented with short sequences of visual and audiovisual stimuli on the screen while their point of gaze in the display area was tracked (120 samples/s). The presentation of the stimuli on the screen and the synchronisation of the stimulus event data and eye-tracking data were controlled by a Python script (https://github.com/infant-cognition-tampere/drop) using Psychopy functions ${ }^{35}$ and a Tobii SDK plug-in. The script includes a graphical user interface that allowed the data collector to follow real-time visualisations of the stimulus presentation and eye-tracking data streams throughout the testing (figure 2). The software was run on Linux operating system (Mint 17) and a Lenovo X260 laptop computer (Lenovo, Morrisville, North Carolina, USA), and was connected to the stimulus display via an HDMI-DVI cable and to the eye tracker via an Ethernet cable and a TRENDnet 4-Port Broadband Router (TRENDnet, Torrance, California, USA). The devices used in the testing were powered by a solar-chargeable battery (GoalZero Yeti 400, NRG Energy, Houston, Texas, USA). A fully charged battery supplied power for a full day of testing.

\section{Procedure and stimuli}

A Sierra Leonian Mende-speaking data collector was trained to perform the eye-tracking assessments. The data collector seated the caregiver holding the infant in a forward-facing baby carrier such that the infant's eyes were at the optimal distance from the eye tracker. Small variation in sitting height was adjusted for by using pillows. The data collector instructed the caregiver to turn their head and eyes to the side $\left(\sim 90^{\circ}\right.$ from the screen $)$ and to avoid looking at the screen during the assessment. The data collector then moved outside the tent laboratory for the duration of testing while constantly monitoring the caregiver and the infant through two monitors. One monitor showed an overall view of the caregiver and the infant. The other monitor showed a visualisation of infant gaze position on the screen.

The test consisted of two sessions that lasted 3-4 min each. These sessions were composed of an alternating sequence of dynamic stimuli, including (1) calibration targets, (2) videos depicting a short ( $5 \mathrm{~s}$ ) or a longer (9-15 s) dyadic social scene with faces and (3) saccade targets. A design in which calibration targets, naturalistic videos of social scenes and saccade targets were presented in an alternating sequence was used to minimise the monotony of the test sessions. The order of the stimuli was the same for all infants: (1) 3-9 calibration targets, (2) a long social scene (1), (3) five saccade targets, (4) a short social scene (1), (5) five saccade targets, (6) a long social scene (1), (7) five saccade targets, (8) a short social scene (1) and (9) five saccade targets. Infants completing the required two sessions saw a total of 6-18 calibration targets, eight blocks of five saccade targets (40 saccade targets in total) and four long and four short social scenes on each visit. Further details of the stimuli are provided below.

While the infants were viewing the stimuli on the screen, their pupil diameter, point of gaze in the display area and position of the eye in a three-dimensional user 
coordinate system were recorded (120 samples/s). If the infant became restless, inattentive or fussy during the procedure, or if the eye-tracking system lost contact with the eyes, the experimenter paused the test and entered the laboratory to administer a break and/or to perform required adjustments (eg, adjusted the infant's position).

\section{Calibration targets}

Calibration targets were short sequences of stimuli which started by the appearance of an audiovisual animation $\left(5.7^{\circ} \times 5.7^{\circ}\right)$ in one of three locations: the centre $\left(0^{\circ}, 0^{\circ}\right)$, bottom-left $\left(-11.4^{\circ},-11.4^{\circ}\right)$ or top-right $\left(11.4^{\circ}, 11.4^{\circ}\right)$ corners of the display area. After the infant looked at the animation or a 3-s timeout value passed, the animation was replaced by an isoluminant white disc $\left(1.3^{\circ} \times 1.3^{\circ}\right)$ for one second, after which the original animation reappeared in the same location and was played for another second. The targets were presented up to two times if less than $75 \%$ of the recorded point of gaze samples were valid for more than one of the targets. Calibration of the raw point-of-gaze estimates was performed using a similarity transformation estimator (https://pypi.org/project/ nudged/) on samples that fell within a $9^{\circ} \times 9^{\circ}$ rectangle surrounding the white discs. The accuracy of the calibrated point-of-gaze estimates was verified by calculating the Euclidean distance of the corrected estimates from the location of the targets at the start of the experiment and half-way through the experiment. Similar comparisons were performed for the raw, uncalibrated point of gaze estimates, and the method that provided the lowest error was used: corrected values were superior for $88 \%$ of infants for visit 1 data and $83 \%$ for visit 2 data.

\section{Saccade targets}

Saccade targets were colourful cartoon animations of common objects (eg, fish, face, mouse, pig, soccer ball or bird), subtending a $5.7^{\circ} \times 5.7^{\circ}$ visual angle. Following a previous study using a similar approach, ${ }^{36}$ the targets were presented one at a time without onset delay so that the first target was always presented in the centre of the screen $\left(0^{\circ}, 0^{\circ}\right)$, and each subsequent target in a new randomly chosen on-screen location $10^{\circ}$ away. After appearing on screen, the animations remained paused at the first frame and the accompanying audio silenced until the infant looked at the animation (ie, the first gaze point overlapping with the target area was recorded) or a 1-s wait period elapsed. The animation was subsequently played for $2500 \mathrm{~ms}$ with an accompanying sound (eg, water bubbling, bird singing or animal sounds, or non-linguistic utterances). The initial stimulus was not included in analysis, as the starting position of the first saccade was unknown. Following the initial stimulus, infants saw a total of five saccade targets in one block, and a total of eight blocks on each visit. Clearly discernible, colourful animations that varied with each presentation were chosen based on infants' known proclivity for novelty and in line with evidence showing superior response rates for this stimulus style. ${ }^{37}$ The stimuli were presented in blocks of five targets in the same order for all participants, but the order of the stimuli was randomised within each block. Additionally, the position of the stimulus was randomly chosen from one of two randomly ordered lists.

\section{Social scenes}

Videos depicting short social scenes were 5-15 s video recordings of two Sierra Leonian Mende-speaking female models. Two of the videos were short $5 \mathrm{~s}$ recordings of two different female models who looked directly at the camera, and thus the infant, and greeted the child in the child's natural language. The other four videos were 9.5-15 s video recordings of four different models again looking at the camera while enacting a short story or a song appropriate for the participants' age range. The videos were taken in various environments (eg, a kitchen, yard or living room area). Infants saw a total of four short videos (two repetitions per video) and four long videos (one presentation per video) on each visit. The videos were similar to those used in previous studies examining infants' fixations to faces. ${ }^{19}$

\section{Measures}

The current analyses focused on SRTs. Analyses of the eyetracking data collected during social scene viewing will be reported separately. SRTs in infants have been variably analysed by using a fixed threshold for eye-movement velocity to detect the onset of a saccadic eye movement, ${ }^{36}$ by parsing the data into periods of saccades and fixations and detecting the latency of the first fixation in the target area, ${ }^{33}$ and by detecting the first recorded point of gaze value in the target area. ${ }^{22}$

For the current study, the method based on the detection of the first recorded point of gaze in the target area was used based on prior work validating this approach for automated SRT analysis in children. ${ }^{22}$ We defined SRTs as the time interval from the onset of the saccade target to the first entry of the gaze into the area of the saccade target. Following the previous validation study using this approach, SRTs were extracted from data that had been filtered with a 15-sample (128 ms) median filter to remove abrupt spikes (figure $3 \mathrm{~A}$ ), and in which the XY-coordinates for the two eyes had been merged by averaging (if both eyes returned a valid data point), or by using the data of one valid eye. Also, a narrow margin $\left(0.9^{\circ}\right)$ was added to the area of the target to accommodate small calibration errors (online supplemental video 1$){ }^{22}$

The extracted SRTs were regarded as valid and retained in the analyses if the following further constraints were met: (1) the starting position of the saccade was controlled so that the gaze moved from the area of the previous saccade target to the area of the new target (ie, the recorded point of gaze was within the area of the previous target for the majority of the period preceding the saccade, excluding a 50-ms transition period), (2) the analysis period did not have consecutive missing samples exceeding $100 \mathrm{~ms}$, (3) a missing sample did not precede the gaze entry to the target area (ie, the exact 


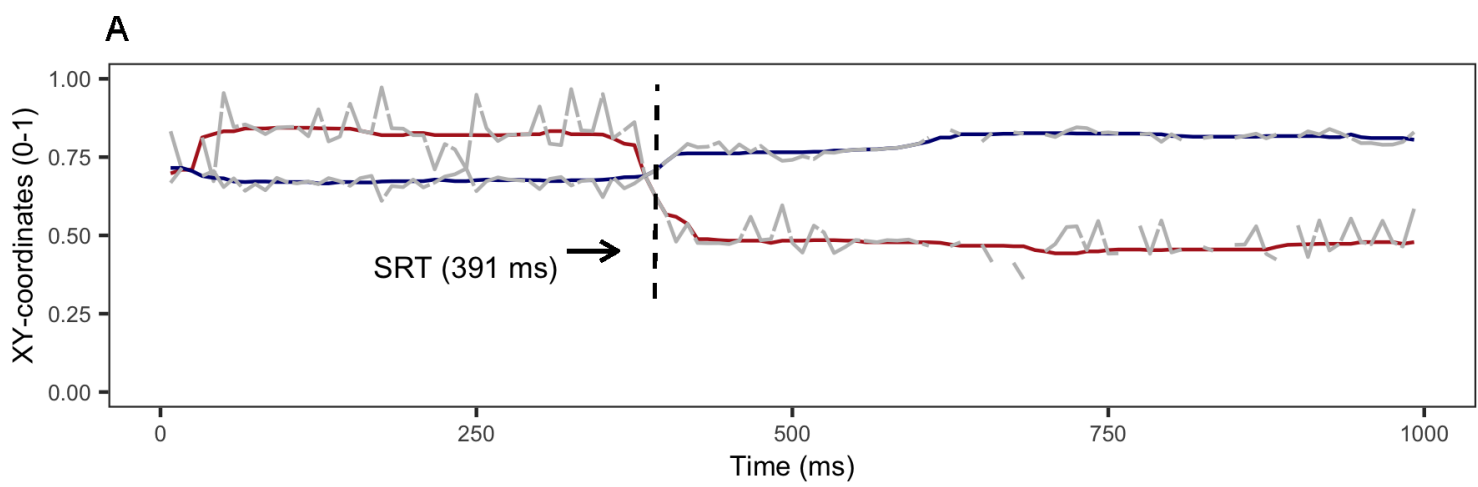

B

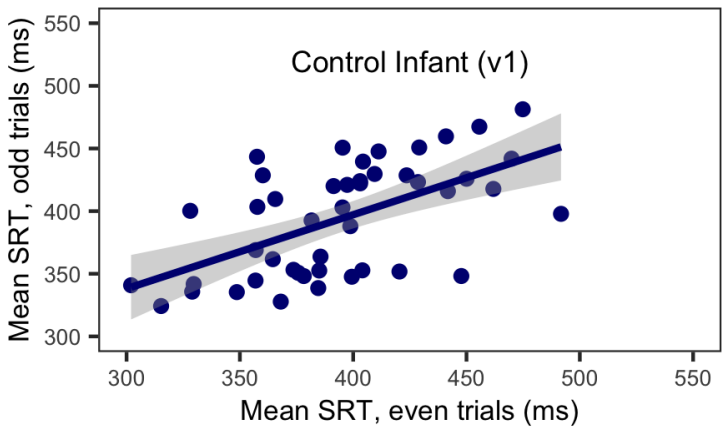

D

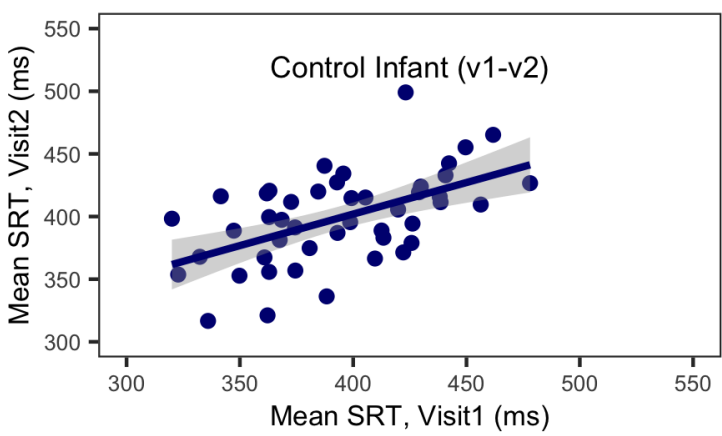

C

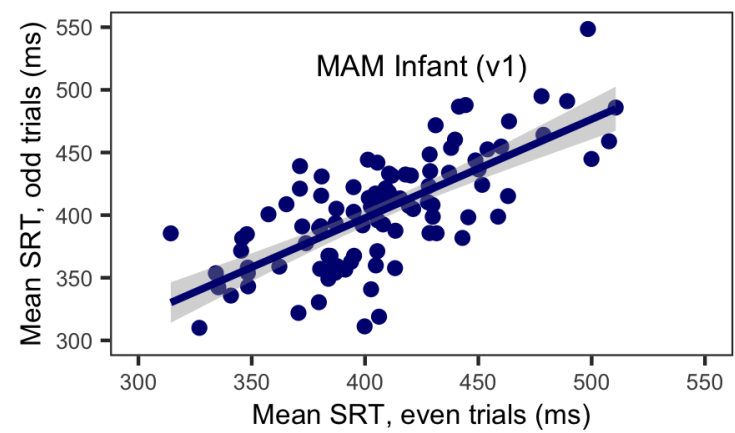

E

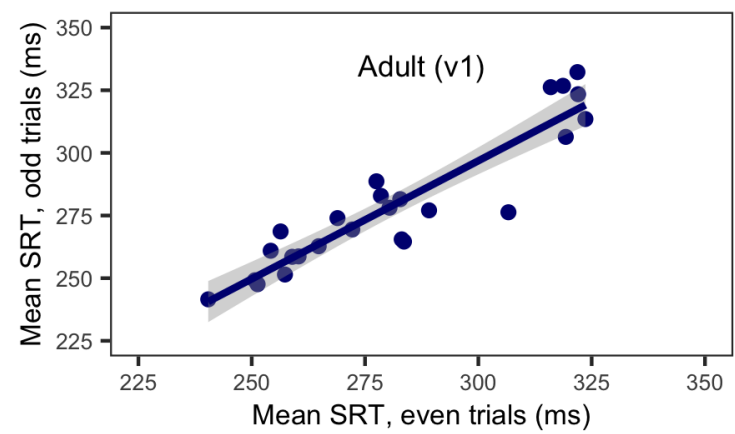

Figure 3 (A) Raw (grey) and median-filtered $X$ (red) and $Y$ (blue) coordinates of the point of gaze on a single trial from one infant. Saccadic reaction times (SRTs) were extracted from the median-filtered data by detecting the first entry of the gaze into the area of the new saccade target. (B-E) Scatterplots showing the consistency of individual participant SRTs across splithalf subsets of trials on visit $1(B, C)$, across the 4-week test-retest interval in the infant control group (D) and across split-half subsets of trials in adults (E). MAM, moderate acute malnutrition.

point of entry was known within the limits of the eyetracking sampling frequency), (4) the SRT fell inside the commonly used expected time window starting $100 \mathrm{~ms}$ after target onset and ending $1000 \mathrm{~ms}$ after target onset. SRTs identified as outliers (2.5 SD from mean SRT) were excluded from statistical analyses, which excluded an additional $1.15 \%$ of all SRTs. These constraints were similar to those used in the previous validation study, ${ }^{22}$ although some parameter values were adjusted to achieve better control the starting position of the saccades and to accommodate the denser spacing of the stimuli in the current study. Further description of these adjustments and results obtained by the previously used parameters is provided in online supplemental methods and analysis and table S2. ${ }^{22}$

\section{Conventional eye-movement testing}

Conventional tests included fixation, saccades and smooth pursuit. ${ }^{38}$ In the tests of visual fixation, a target (toy) was presented for $4 \mathrm{~s}$ in an alternating sequence in the child's frontal, lower left, upper left, lower right and upper right visual field. The child's response (fixation) was scored by using a 3-point scale where 0 indicated no fixation, 0.5 partial and 1 complete fixation of the target. In the saccade and smooth pursuit tests, the first target (a colourful rattle) was presented in the child's field of vision at eye level, centred with respect to the child's line of gaze and at approximately $50 \mathrm{~cm}$ from the child's eyes. When the child fixated on the target, a second similar target was presented to the child's lateral field, at the same level as the first target and approximately $50 \mathrm{~cm}$ to 
Table 1 Baseline characteristics of study infants

\begin{tabular}{|c|c|c|c|}
\hline & Control & MAM & \\
\hline & $\mathrm{n}=46$ & $n=96$ & $P$ value \\
\hline Female, n (\%) & $24(52)$ & $63(66)$ & 0.12 \\
\hline Age, months* & $9(7.2,11.7)$ & $8.4(7,11.9)$ & 0.11 \\
\hline Length, cm & $67.6 \pm 3.3 \dagger$ & $64.1 \pm 3$ & $<0.001$ \\
\hline Weight, kg & $7.7 \pm 1.1$ & $6.1 \pm 0.6$ & $<0.001$ \\
\hline MUAC, $\mathrm{cm}^{\star}$ & $13.6(12.6,15.8)$ & $12.0(11.5,12.5)$ & $<0.001$ \\
\hline $\begin{array}{l}\text { Head } \\
\text { circumference, } \mathrm{cm}\end{array}$ & $43.6 \pm 1.4$ & $42 \pm 1.4$ & $<0.001$ \\
\hline WHZ & $-0.2 \pm 1 \dagger$ & $-1.5 \pm 0.6$ & $<0.001$ \\
\hline HAZ & $-1.4 \pm 1 \dagger$ & $-2.7 \pm 1.1$ & $<0.001$ \\
\hline WAZ & $-1 \pm 1$ & $-2.8 \pm 0.7$ & $<0.001$ \\
\hline $\begin{array}{l}\text { Caregiver literacy, } \\
\text { n (\%) }\end{array}$ & $6(13)$ & $13(13.5)$ & 1.0 \\
\hline \multicolumn{4}{|l|}{ Food group, n (\%) } \\
\hline $\begin{array}{l}\mathrm{SC}+\text { with } \\
\text { amylase }\end{array}$ & & $26(27)$ & \\
\hline RUSF & & $28(29)$ & \\
\hline $\mathrm{CSB}+$ & & $29(30)$ & \\
\hline CSWB & & $13(14)$ & \\
\hline
\end{tabular}

All values are means \pm SD unless otherwise indicated. - indicate data are not applicable. Means were compared using independent samples t-test unless otherwise indicated. Proportions were compared using $\chi^{2}$ test.

*Median (min, max), compared with Mann-Whitney U test. $\dagger \mathrm{t}=45$.

CSB+, corn-soy blend plus; CSWB, corn-soy whey blend; HAZ, height-for-age z-score; MAM, moderate acute malnutrition; MUAC, mid-upper arm circumference; RUSF, ready-to-use-supplementary food; SC+, super cereal plus; WAZ, weight-for-age z-score; WHZ, weight-for-height z-score.

the left or right of the first target. After the child shifted gaze from the first (central) to the second (lateral) target, the lateral target was moved toward the central target. The procedure was repeated twice for both directions. Saccades from the central to the lateral target and smooth pursuit eye movements following the moving target were scored by using a 3-point scale, where 0 indicated absence of saccade or smooth pursuit, 0.5 partial and 1 complete saccade or smooth pursuit. For statistical analysis, the data from the oculomotor testing were aggregated within tasks and subsequently averaged across tasks to a composite measure of oculomotor function.

\section{Adult control testing}

The stability of the eye-tracking measures in infants may be affected by 'instrument noise' (ie, limitations of the employed test and analysis setup in reliably detecting and quantifying the timing of saccadic eye movements) and/or more genuine trial-by-trial variability in the actual behavioural responses of infants. ${ }^{39}$ To examine major sources of instrument noise in the current setup, we tested a reference group of adult observers with the same eye-tracking setting methodology. Mean SRTs are known to have high within session stability in adults, with Spearman rank correlation for split-half subsets of the data ranging from 0.85 to $0.95 .{ }^{40}$ The data were analysed using the same pre-processing scripts and parameters as those used with infants.

\section{Anthropometric and survey procedures}

Anthropometry in infants was measured every 2 weeks. Nude weight was measured to the nearest $5 \mathrm{~g}$, recumbent length was measured using a rigid length board in duplicate to the nearest $0.1 \mathrm{~cm}$, and MUAC was measured using a standard insertion tape applied to the left arm and to the nearest $0.1 \mathrm{~cm}$. A socioeconomic survey was conducted with each caretaker. Anthropometric and socioeconomic data were collected on standardised clinic cards and entered into a password protected KoBoCollect database. Anthropometric indices were calculated using WHO Child Growth Standards (Anthro V.3.1, WHO, Geneva).

\section{Statistical testing}

For baseline characteristics in the two cohorts, categorical variables were summarised with $\%$ and compared using $\chi^{2}$, while continuous variables were assessed first for conformance to normality by observing their distribution. If normality was approximated, values were compared using Student's t-test; otherwise, values were compared using Mann-Whitney $\mathrm{U}$ test. $\mathrm{P}$ values $<0.05$ were classified as statistically significant.

The feasibility and measurement properties of eyetracking tests were examined by calculating descriptive statistics for calibration error, number of valid test trials available for the estimation of mean SRTs and the stability of SRTs. To examine the stability of SRTs (ie, agreement of measures across separate tests), we calculated intraclass correlation coefficients (ICCs) with a two-way random effects model for (1) means computed by dividing the data from visit 1 into two subsets based on odd and even numbered trials and (2) means from visits 1 and 2 (using data from the non-MAM control group). We also calculated the SD of the differences in the measures between visits 1 and $2\left(\mathrm{SD}_{\text {Difference }}\right)$, as well as an estimate of the smallest detectable difference in SRTs for individual infants $\left( \pm 1.96 * \mathrm{SD}_{\text {Difference }}\right)$.

Change in SRT was calculated as week 4 SRT minus baseline SRT. Baseline SRT and change in SRT were compared between the two cohorts using independent samples t-test as well as ordinary least squares (OLS) regression with adjustment for age and sex. Change in SRT was also compared by supplement received using both independent samples t-test and OLS regression adjusted for age and sex. Analyses were performed using R (V.4.0.3) and SPSS (V.26.0, IBM, Armonk, New York, USA). ${ }^{41}$

\section{RESULTS}

\section{Sample description}

Of the 198 infants enrolled in the substudy, 149 participated in the eye-tracking assessments at visit 1 (baseline) 


\begin{tabular}{|c|c|c|c|}
\hline Measure & Control & MAM & $P$ value \\
\hline Calibr trials $\mathrm{V} 1^{*}$ & $\mathrm{M}=5.0(\mathrm{SD}=1.3)$ & $M=5.2(S D=1.3)$ & 0.34 \\
\hline $\mathrm{X}$-axis calibr error $\mathrm{V} 1$ & $\mathrm{M}=0.7^{\circ}(\max =1.9)$ & $M=0.8^{\circ}(\max =2.1)$ & 0.58 \\
\hline $\mathrm{X}$-axis calibr error V2 & $\mathrm{M}=0.7^{\circ}(\max =1.9)$ & $M=0.6^{\circ}(\max =3.7)$ & 0.29 \\
\hline Y-axis calibr error V2 & $M=1.1^{\circ}(\max =3.8)$ & $M=1.1^{\circ}(\max =3.6)$ & 0.68 \\
\hline SRT valid trials V1 & $M=19$ (range 10-31) & $M=22$ (range $10-38$ ) & 0.03 \\
\hline SRT valid trials V2 & $\mathrm{M}=22$ (range 10-33) & $\mathrm{M}=23$ (range 11-37) & 0.46 \\
\hline SRT response $\mathrm{p} V 1$ & $M=99.5 \%$ (range $88.9-100$ ) & $M=99.3 \%$ (range $81.3-100.0$ ) & 0.58 \\
\hline SRT response p V2 & $\mathrm{M}=99.4 \%$ (range 94.7-100.0) & $\mathrm{M}=99.2 \%$ (range 92.8-100.0) & 0.72 \\
\hline
\end{tabular}

${ }^{*}$ Calibration data were missing for visit 1 ( 4 controls and 4 infants with MAM) and visit 2 ( 1 control and 5 MAM).

ICC, intraclass correlation coefficient; MAM, moderate acute malnutrition; response $p$, probability of a response to the saccade target on valid trials; SRT, saccadic reaction time; V1, visit 1; V2, visit 2.

and visit 2 (4 weeks post-enrolment); 11 did not participate in visit 2 testing and thus were excluded (figure 1). Of those who underwent testing, 3 infants $(2 \%)$ were excluded due to discovered non-eligibility (neurological history), and 38 infants (19\%) were excluded for insufficient number of valid eye-tracking test trials from visits 1 or $2(<10$ trials/visit, figure 1$)$. Four infants $(7 \%)$ in the control group developed MAM by visit 2 and were thus excluded. Baseline characteristics of the 142 children show significant differences across all anthropometric criteria between MAM and control children (table 1).

Thirty participants were enrolled in the adult control group and tested with the same paradigm as that used with infants. Twenty-five were retained in the analyses: 17 $(68 \%)$ were women, with a mean age of 22 (range 18-31). One adult was excluded due to discovered non-eligibility (age $>40$ ), three for lost screening or eye-tracking data and one for $<10$ valid test trials.

\section{Eye-tracking tests}

Measurement properties

In both MAM and control infants, the average number of interruptions per session was $<1$. Descriptive statistics for calibration error, number of valid trials, probability of response to saccade targets and the within-session and between-session stability of the mean SRTs are provided in table 2. Mean calibration error in the estimates of the point of gaze (ie, distance from the returned value to the actual location of a calibration target) ranged from $0.68^{\circ}$ to $1.14^{\circ}$ (online supplemental figure 1 ), with no significant differences between the MAM and non-MAM control groups. MAM children had more valid trials at baseline than non-MAM controls $(p=0.03)$, but there were no differences between the groups in the postenrolment assessment. Mean SRTs had moderate stability within visits (ICCs 0.60-0.69) and across the 4-week test-retest interval $(0.53$, figure $3 \mathrm{~B}-\mathrm{D})$. Using data from the test-retest comparisons in the control group with no predicted change in the behaviour, the estimated SE of SRT means was $25 \mathrm{~ms}$, and the smallest detectable change in individual subject SRT $68 \mathrm{~ms}$.

In the adult control group, mean calibration error in the estimates of the point of gaze was $0.9^{\circ}\left(\max =2.1^{\circ}\right)$ and $1.14^{\circ}\left(\max =3.3^{\circ}\right)$ horizontally and vertically, respectively. Adults contributed on average 32 valid trials for the analysis of mean SRTs (range 11-39). The rank order correlation of the SRTs for split-half subsets was high (Spearman $\mathrm{r}=0.87$ ), and the absolute agreement of the two mean SRTs was also high (ICC=0.92, $95 \%$ CI 0.83 to 0.97 , figure $3 \mathrm{E}$ ).

\section{Association with nutritional status}

The MAM group displayed a trend to higher unadjusted and adjusted baseline SRT compared with controls (table 3). SRT shortened in the MAM group after 4 weeks compared with controls (table 3). Changes in SRTs for individual participants are shown in figure 4. Comparison of change in SRT in infants by supplementary food group revealed a shortening of SRT in each food group compared with change in control, with the exception of CSWB (online supplemental table 3).

\section{Conventional oculomotor assessment}

Complete data on the conventional oculomotor assessment were available for 87 MAM children and 41 controls at visit 1 and 63 MAM and 24 controls at visit 2. Complete data from both visits were available for 57 MAM children and 19 controls.

Distribution of responses to individual observational tests assessing saccades, fixations and smooth pursuit of colourful toy objects in a naturalistic interaction situation are shown in online supplemental figure 2. The mean 
Table 3 Differences in baseline SRT and change in SRT in children with MAM compared with controls in unadjusted and adjusted analyses

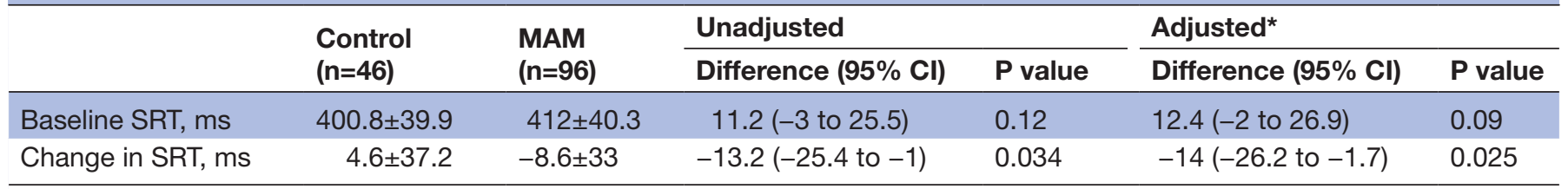

Data are mean \pm SD and mean difference $(95 \% \mathrm{Cl})$. Independent samples t-test was used for unadjusted comparisons. Linear regression was used for adjusted comparisons.

${ }^{*}$ Adjusted for age and sex.

MAM, moderate acute malnutrition; SRT, saccade reaction time.

total scores did not differ between the MAM and control groups at baseline (table 4). There were also no differences in changes scores between the MAM children and the controls.

\section{DISCUSSION}

Eye-tracking technologies have been widely used to examine infant attention and cognitive functions in high-resource laboratory settings in Western countries. Recently, the use of this method has also been expanded to low-income countries. ${ }^{23}{ }^{25-30}$ This report documents a novel use of eye-tracking in the assessment of neurocognition of infants classified as moderately wasted (experiencing MAM) before and after supplementary feeding in settings that have very few facilities and thus represent the circumstances of many resource-limited communities in Sub-Saharan Africa.

The key metric of visual orienting (mean SRT) was obtained for most infants at the first attempt of testing at two time points. Mean SRTs showed moderate levels
A

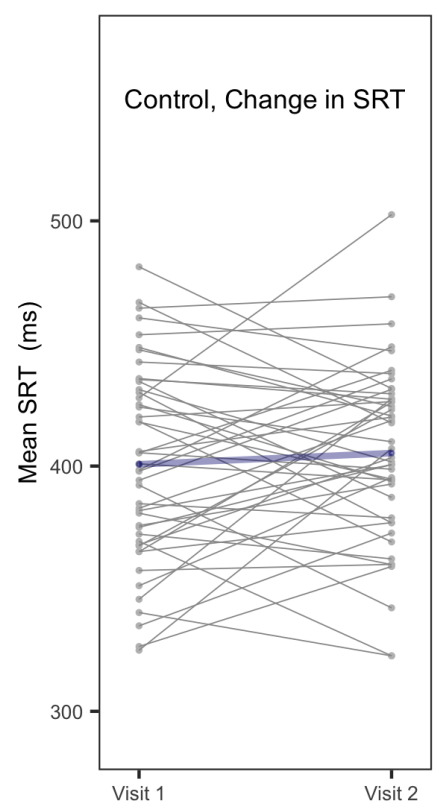

B

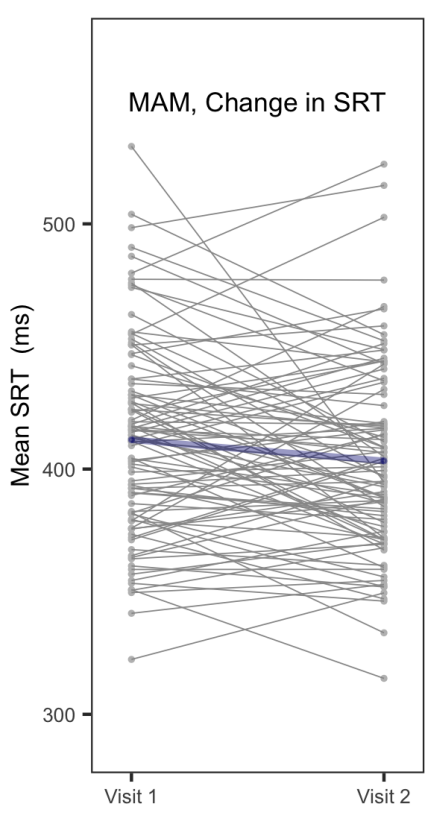

Figure 4 Mean saccadic reaction times (SRTs) by visit for individual infants (grey lines). Group mean is shown by the blue line. MAM, moderate acute malnutrition. of within-observer consistency. Relatively high data and participant attrition rates and the presence of withinsubject variability in performance metrics are common findings in studies of infant cognition. The observed levels of data attrition and absolute agreement of mean SRTs within sessions and over a 4-week test-retest interval were within the range of the values reported in previous studies in controlled laboratory environments in infants $(\mathrm{r}=0.53-0.65) .{ }^{33}$ The high precision of the estimates in the adult control group suggests that the comparatively lower stability seen in infants is not due to instrument noise, but rather reflects inherent variability in infant behaviour. Together, these results suggest that there are no major barriers for wider use of eye-tracking in the field in underserved settings. However, the fact that the measures had only moderate within-subject stability in infants indicates that, as currently implemented, eye-tracking measures of visual orienting have more utility in the detection of group level differences (eg, intervention effects) than in the monitoring of neurocognitive changes and recovery in individual infants.

Our data revealed a trend toward longer SRT in infants with acute malnutrition, though the 95\% CI included negligible (2 ms shorter) and much larger (27 ms longer) differences. This result may reflect that acute malnutrition is but one of many factors that impact cognition, as all participants were from environments that are disadvantaged by many measures. The mean SRTs in the current study were clearly slower than the mean SRTs typically found for infants in this age range. Direct comparisons are complicated by procedural differences between studies, but the potential difference is large: mean SRTs were 400-410 ms in the current study and a recently published study in rural Malawi, ${ }^{30}$ whereas the corresponding values in previous studies in high-resource settings have ranged from 270 to 375 ms. ${ }^{13} 314243$ Further research is thus needed to determine the degree and significance of the effects of both acute and chronic malnutrition as well as other poverty-related risk factors on neurocognitive development.

As a preliminary demonstration of the potential neurocognitive benefits of supplementary feeding in MAM, our results showed a significant improvement in SRT in the MAM group after 4 weeks of supplementary feeding. This change in SRT was, however, small 
Table 4 Aggregate scores in the coventional tests of oculomotor function in children with MAM compared with controls

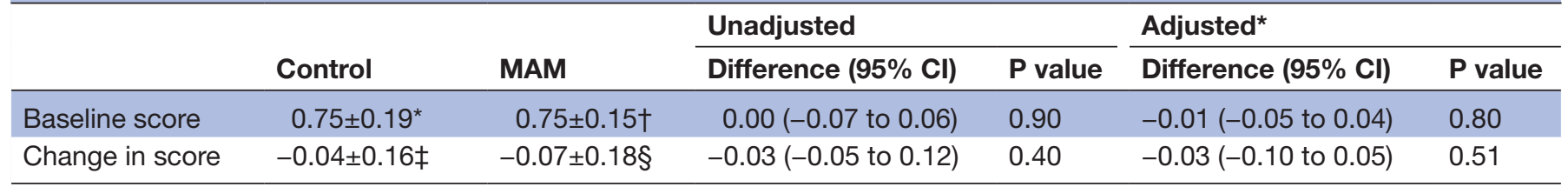

Data are mean \pm SD and mean difference $(95 \% \mathrm{Cl})$. Independent samples t-test was used for unadjusted comparisons. Linear regression was used for adjusted comparisons.

${ }^{*} \mathrm{n}=41$.

$\dagger \mathrm{t}=87$.

$\ddagger \mathrm{n}=24$.

$\S \mathrm{n}=63$.

|Adjusted for age and sex.

MAM, moderate acute malnutrition; SRT, saccade reaction time.

and only evident at the group level. Changes in SRTs showed high variability in individual infants in both the MAM and control groups. The interpretation of the group-level change as indication of improvements in neurocognitive function is supported by previous data showing that specific nutritional supplements result in similar changes in SRT in high resource settings, ${ }^{31}$ by the established status of faster orienting as a predictive marker of cognitive function, ${ }^{17} 18$ and, lastly, by the apparent functional benefits that come from faster visual orienting. There were also no indications in the data that the differential change in SRT between the MAM and control groups reflected secondary effects arising from the targeted behaviours becoming more 'measurable' between the two visits (eg, the number of valid trials being disproportionally low in the MAM group on the first visit). Small sample size limited the ability to assess differential effects of the four food groups from the parent study, but the results showed a consistent effect of improvement in SRT, with the exception of CSWB. It is unclear why children receiving CSWB did not demonstrate the same effect, though it is notable that these foods were not designed for neurocognitive benefit, but rather for anthropometric growth. Despite this, the possibility remains that the size of the improvements in the MAM group may change by supplement type, and in, particular, by the use of more neurocognitively targeted nutrients.

Our study has multiple limitations. First, it is a cohort study with age-matched controls chosen as a convenience sample and so remains open to confounding factors impacting the results and interpretation. Second, the saccade reaction time methodology requires a minimum number of successful tests, which led to the exclusion of approximately $25 \%$ of the initial sample. It is possible these children systematically differed from those included and thus might have altered results. Third, we used different saccade targets on every trial to maximise the interest value of the stimuli. A trade-off of this decision was that some of the variability in SRTs may have resulted from the varying physical and semantic properties of the stimuli. Fourth, while an improvement in SRT was found at group level, the presence of this effect was small and highly variable between individual infants. Fifth, this study was performed in one district in Sierra Leone and so the degree of generalisability is uncertain. Additionally, the novel method limits comparisons to testing done previously in other settings, and so the significance of the results remains unclear.

The study also has multiple strengths. Testing each child twice allowed for assessment of both precision in the testing and changes over time. The control infant group was appropriately age-matched and significantly different than the MAM children across all anthropometrics. The adult control group allowed for parsing the contributions of methodology and individual factors to variability.

Taken together, this study demonstrates that visual orienting can be successfully assessed by eye-tracking in infants in resource-poor settings. This technique offers the potential to investigate the acute cognitive dimensions of malnutrition and its myriad effects on development robustly. The combination of its lack of relation to standard anthropometrics and its apparent responsiveness to supplemental feeding in acute malnutrition suggest it might be shedding light on a different aspect of malnutrition.

\section{Author affiliations}

${ }^{1}$ Department of Psychology and Speech-Language Pathology, University of Turku, Turku, Finland

${ }^{2}$ Project Peanut Butter, Freetown, Sierra Leone

${ }^{3}$ Department of Pediatrics, Washington University in St Louis School of Medicine, St Louis, Missouri, USA

${ }^{4}$ Department of Internal Medicine, Washington University in St Louis School of Medicine, St Louis, Missouri, USA

${ }^{5}$ Friedman School of Nutrition Science and Policy, Tufts University, Boston, Massachusetts, USA

${ }^{6}$ Food and Nutrition, Sierra Leone Ministry of Health and Sanitation, Freetown, Sierra Leone

Twitter D Taylor Hendrixson @DTHENDRIXSONMD and Patrick Webb @ DrPatrickWebb

Acknowledgements The authors thank the participants and their caregivers for their dedication to the program and the Sierra Leone Ministry of Health and Sanitation, Nutrition Directorate for their valuable expertise. 
Contributors JML, KS, DTH and MJM wrote the first draft of this manuscript. JML, ASK and MJM conceptualised the study. DTH, ASK, SG and MJM selected the sites. JML, JWB and MJM developed and adapted the eye-tracking software and equipment. JML, JWB, DTH and MJM developed the trial protocol. JML, DTH, SG, BLR, PW and MJM provided supervision of the trial. JML, JWB, CG and DTH performed data collection in the field. JML, KS and MJM performed statistical analysis and interpreted the data. MJM is guarantor of the trial, data and manuscript. All authors read and approved the final manuscript. MJM affirms that the manuscript is an accurate account of the study being reported and that no important aspects of the study have been omitted.

Funding This work was supported by USAID, grant AID-OAA-C-16-00020. JML, SG, CG, PW and MJM received research support from USAID AID-OAA-C-16-00020. DTH received support from National Institute of Diabetes and Digestive and Kidney Diseases under Award Number T32 DK077653. KS received support from the Knowlton Foundation at Washington University in St Louis.

Competing interests None declared.

Patient consent for publication Not applicable.

Ethics approval The four foods trial was approved by Tufts University Human Studies Committee (ID 12313), the Washington University Human Studies Committee (ID 201610030) and the Sierra Leone Ethics and Scientific Review Committee. The study in which adult controls were recruited was approved by the Washington University Human Studies Committee and the Sierra Leone Ethics and Scientific Review Committee.

Provenance and peer review Not commissioned; externally peer reviewed.

Data availability statement Data are available in a public, open access repository. Data described in the manuscript, code book and analytic code were submitted to USAID's open repository and will be made publicly and freely available without restriction soon at https://data.usaid.gov.

Supplemental material This content has been supplied by the author(s). It has not been vetted by BMJ Publishing Group Limited (BMJ) and may not have been peer-reviewed. Any opinions or recommendations discussed are solely those of the author(s) and are not endorsed by BMJ. BMJ disclaims all liability and responsibility arising from any reliance placed on the content. Where the content includes any translated material, BMJ does not warrant the accuracy and reliability of the translations (including but not limited to local regulations, clinical guidelines, terminology, drug names and drug dosages), and is not responsible for any error and/or omissions arising from translation and adaptation or otherwise.

Open access This is an open access article distributed in accordance with the Creative Commons Attribution Non Commercial (CC BY-NC 4.0) license, which permits others to distribute, remix, adapt, build upon this work non-commercially, and license their derivative works on different terms, provided the original work is properly cited, appropriate credit is given, any changes made indicated, and the use is non-commercial. See: http://creativecommons.org/licenses/by-nc/4.0/.

\section{ORCID iDs}

Kevin Stephenson http://orcid.org/0000-0003-4284-1062

D Taylor Hendrixson http://orcid.org/0000-0003-0627-3571

Stacy Griswold http://orcid.org/0000-0001-7572-3316

Patrick Webb http://orcid.org/0000-0002-9857-3354

\section{REFERENCES}

1 Prado EL, Dewey KG. Nutrition and brain development in early life. Nutr Rev 2014;72:267-84.

2 Nelson CA, Zeanah CH, Fox NA, et al. Cognitive recovery in socially deprived young children: the Bucharest early intervention project. Science 2007;318:1937-40.

3 Georgieff MK. Nutrition and the developing brain: nutrient priorities and measurement. Am J Clin Nutr 2007;85:614S-20.

4 Walker SP, Wachs TD, Grantham-McGregor S, et al. Inequality in early childhood: risk and protective factors for early child development. Lancet 2011;378:1325-38.

5 Waber DP, Bryce CP, Girard JM, et al. Impaired IQ and academic skills in adults who experienced moderate to severe infantile malnutrition: a 40-year study. Nutr Neurosci 2014;17:58-64.

6 Galler JR, Bryce CP, Waber DP, et al. Infant malnutrition predicts conduct problems in adolescents. Nutr Neurosci 2012;15:186-92.

7 Galler JR, Bryce CP, Zichlin ML, et al. Malnutrition in the first year of life and personality at age 40. J Child Psychol Psychiatry 2013;54:911-9.
8 Hsieh J-C, Liu L, Zeilani M, et al. High-Oleic ready-to-use therapeutic food maintains docosahexaenoic acid status in severe malnutrition. $J$ Pediatr Gastroenterol Nutr 2015;61:138-43.

9 Jandó G, Mikó-Baráth E, Markó K, et al. Early-onset binocularity in preterm infants reveals experience-dependent visual development in humans. Proc Natl Acad Sci U S A 2012;109:11049-52.

10 Frank MC, Vul E, Saxe R. Measuring the development of social attention using Free-Viewing. Infancy 2012;17:355-75.

11 Arcaro MJ, Schade PF, Vincent JL, et al. Seeing faces is necessary for face-domain formation. Nat Neurosci 2017;20:1404-12.

12 Hunnius S, Geuze RH, van Geert P. Associations between the developmental trajectories of visual scanning and disengagement of attention in infants. Infant Behav Dev 2006;29:108-25.

13 Kulke L, Atkinson J, Braddick O. Automatic detection of attention shifts in infancy: eye tracking in the fixation shift paradigm. PLoS One 2015;10:e0142505.

14 Canfield RL, Wilken J, Schmerl L, et al. Age-related change and stability of individual differences in infant saccade reaction time. Infant Behav Dev 1995;18:351-8.

15 Rose SA, Feldman JF, Jankowski JJ. The building blocks of cognition. J Pediatr 2003;143:54-61.

16 Pearson BC, Armitage KR, Horner CWM, et al. Saccadometry: the possible application of latency distribution measurement for monitoring concussion. Br J Sports Med 2007;41:610-2.

17 Dougherty TM, Haith MM. Infant expectations and reaction time as predictors of childhood speed of processing and IQ. Dev Psychol 1997;33:146-55.

18 Rose SA, Feldman JF, Jankowski JJ. Implications of infant cognition for executive functions at age 11. Psychol Sci 2012;23:1345-55.

19 Jones W, Klin A. Attention to eyes is present but in decline in 2-6-month-old infants later diagnosed with autism. Nature 2013;504:427-31.

20 Peltola MJ, Yrttiaho S, Leppänen JM. Infants' attention bias to faces as an early marker of social development. Dev Sci 2018;21:e12687.

21 Jones PR, Kalwarowsky S, Atkinson J, et al. Automated measurement of resolution acuity in infants using remote eyetracking. Invest Ophthalmol Vis Sci 2014;55:8102-10.

22 Leppänen JM, Forssman L, Kaatiala J, et al. Widely applicable MATLAB routines for automated analysis of saccadic reaction times. Behav Res Methods 2015;47:538-48.

23 Forssman L, Ashorn P, Ashorn U, et al. Eye-tracking-based assessment of cognitive function in low-resource settings. Arch Dis Child 2017;102:301.1-2.

24 Pyykkö J, Ashorn U, Chilora E, et al. Associations between individual variations in visual attention at 9 months and behavioral competencies at 18 months in rural Malawi. PLoS One 2020;15:e0239613

25 Katus L, Hayes NJ, Mason L, et al. Implementing neuroimaging and eye tracking methods to assess neurocognitive development of young infants in low- and middle-income countries. Gates Open Res 2019;3:1113.

26 Pyykkö J, Forssman L, Maleta K, et al. Early development of visual attention in infants in rural Malawi. Dev Sci 2019;22:e12761.

27 Boivin MJ, Weiss J, Chhaya R, et al. The feasibility of automated eye tracking with the early childhood vigilance test of attention in younger HIV-exposed Ugandan children. Neuropsychology 2017;31:525-34.

28 Chhaya R, Weiss J, Seffren V, et al. The feasibility of an automated eye-tracking-modified Fagan test of memory for human faces in younger Ugandan HIV-exposed children. Child Neuropsychol 2018;24:686-701.

29 Hernik M, Broesch T. Infant gaze following depends on communicative signals: an eye-tracking study of 5- to 7-month-olds in Vanuatu. Dev Sci 2019;22:e12779.

30 Prado EL, Maleta K, Caswell BL, et al. Early child development outcomes of a randomized trial providing 1 egg per day to children age 6 to 15 months in Malawi. J Nutr 2020;150:1933-42.

31 Caudill MA, Strupp BJ, Muscalu L, et al. Maternal choline supplementation during the third trimester of pregnancy improves infant information processing speed: a randomized, double-blind, controlled feeding study. Faseb J 2018;32:2172-80.

32 Local Burden of Disease Child Growth Failure Collaborators. Mapping child growth failure across low- and middle-income countries. Nature 2020;577:231-4.

33 Cousijn J, Hessels RS, Van der Stigchel S, et al. Evaluation of psychometric properties of the gap-overlap task in 10-month-old infants. Infancy 2017;22:571-9.

34 Griswold SP, Langlois BK, Shen Y, et al. Effectiveness and costeffectiveness of 4 supplementary foods for treating moderate acute malnutrition: results from a cluster-randomized intervention trial in Sierra Leone. Am J Clin Nutr 2021;114:973-85. 
35 Peirce JW. PsychoPy-Psychophysics software in Python. J Neurosci Methods 2007;162:8-13.

36 Alahyane N, Lemoine-Lardennois C, Tailhefer C, et al. Development and learning of saccadic eye movements in 7- to 42-month-old children. $J$ Vis 2016;16:6.

37 Irving EL, González EG, Lillakas L, et al. Effect of stimulus type on the eye movements of children. Invest Ophthalmol Vis Sci 2011;52:658-64.

38 Olsson M, Teär Fahnehjelm K, Rydberg A, et al. Ocular motor score (OMS): a clinical tool to evaluating ocular motor functions in children. intrarater and inter-rater agreement. Acta Ophthalmol 2015;93:444-9.

39 Klein C, Fischer B. Instrumental and test-retest reliability of saccadic measures. Biol Psychol 2005;68:201-13.
40 Bargary G, Bosten JM, Goodbourn PT, et al. Individual differences in human eye movements: an oculomotor signature? Vision Res 2017;141:157-69.

$41 \mathrm{R}$ : A language and environment for statistical computing [program]. Vienna, Austria 2014.

42 Wass SV, Smith TJ. Individual differences in infant oculomotor behavior during the viewing of complex naturalistic scenes. Infancy 2014;19:352-84.

43 Rose SA, Feldman JF, Jankowski JJ, et al. A longitudinal study of visual expectation and reaction time in the first year of life. Child Dev 2002;73:47-61. 